\title{
DIAGNÓSTICO DIFERENCIAL DE LESIONES CEREBRALES CON REALCE EN ANILLO EN TOMOGRAFÍA COMPUTARIZADA Y RESONANCIA MAGNÉTICA
}

\section{DIFFERENTIAL DIAGNOSIS OF BRAIN INJURY WITH BOOST RING IN COMPUTED TOMOGRAPHY AND MAGNETIC RESONANCE IMAGING}

\section{TÍTULO CORTO: DIAGNÓSTICO DIFERENCIAL DE LESIONES CEREBRALES}

Vicente de Jesús Aljure', Edder Armando Pulido-Arias², Jeffer Alexander Rodríguez-Monroy², María Natalia Rodríguez-Mateus ${ }^{2}$, Jhon Alejandro Ramos-Hernández ${ }^{2}$

Recibido en junio 12 de 2015

Aceptado en abril 15 de 2016

\section{RESUMEN}

La tomografía computarizada y la resonancia magnética nuclear son técnicas imagenológicas de diagnóstico médico que ofrecen información excelente de diferentes procesos infecciosos, traumáticos o tumorales de cualquier parte del cuerpo humano. Con ellos se permitió la identificación de innumerables patologías y patrones característicos de las mismas, siendo una de ellas, el realce en anillo en el Sistema Nervioso Central. Numerosas patologías presentan este patrón imagenológico: Glioblastoma multiforme, síndromes desmielinizantes, metástasis, abscesos cerebrales, toxoplasmosis cerebral, neurocisticercosis, linfomas, entre otros. El objetivo del presente artículo es brindar una revisión general de las patologías más frecuentes en los servicios de imagenología, permitiendo su correlación con las características clínicas y brindando una ayuda al clínico que para establecer un diagnóstico adecuado.

Palabras clave: Neuroimagen; Diagnóstico Diferencial; Encefalopatías

\section{ABSTRACT}

Computed tomography and magnetic resonance imaging are medical diagnostic imaging techniques that provide excellent information from different infectious, traumatic or tumor processes anywhere in the body. It is identifying with them many diseases and characteristic patterns of them, one of them the ring enhancement in the central nervous system allowed. Many diseases have this imaging pattern: Glioblastoma multiforme, demyelinating syndromes, metastases, brain abscesses, cerebral toxoplasmosis, neurocysticercosis, lymphomas, and others. The objective of this article is to provide an overview of the most common diseases in imaging services, allowing its correlation with clinical features and providing aid to the clinician to establish a proper diagnosis.

Keywords: Neuroimaging; Diagnosis Differential; Brain Diseases

1. Médico, Especialista en Radiología e Imágenes Diagnósticas, Universidad de Zulia, Venezuela. Docente de Medicina Interna en Radiología e Imágenes Diagnosticas de la Universidad Pedagógica Tecnológica de Colombia, Colombia. Correo: vicentealjure@gmail.com

2. Estudiante de IX semestre de Medicina de la Universidad Pedagógica y Tecnológica de Colombia, Colombia. Correo: edd9408@hotmail.com; alex496@hotmail.es; Natalia-2831@hotmail.com; alejo_5466@hotmail.com 


\section{INTRODUCCIÓN}

$\mathrm{L}$ a Tomografía Computarizada (TC) y la Resonancia Magnética nuclear (RM) son técnicas imagenológicas de diagnóstico médico que ofrecen información valiosa de procesos infecciosos, traumáticos o tumorales de cualquier parte del cuerpo, lo cual permite una toma de decisiones precisa $^{1-3}$. Con estos métodos, se permite la identificación de patrones imagenológicos característicos de las mismas, siendo una de ellas, el realce en anillo en el Sistema Nervioso Central (SNC) ${ }^{4}$.

El patrón de realce en anillo se denomina a lesiones que presentan una cavidad central, homogénea o heterogénea, con un borde regular o irregular, de diferente espesor, y que puede reforzarse tras la administración de medio de contraste. En base a sus características acompañantes se puede sospechar diferentes patologías ${ }^{5}$, siendo de vital importancia de su identificación ya cada una de ellas representa un alto riesgo para el paciente, y de su oportuno diagnóstico y adecuado tratamiento dependerá la gravedad de las secuelas dejadas por estas. Siendo el sistema nervioso uno de los tejidos más frágiles y cuyos daños se generan en breves periodos de tiempo, las enfermedades que se desarrollan en el mismo tienen repercusiones importantes sobre la calidad de vida de quien las padece ${ }^{1,3,5}$; por tal motivo su identificación oportuna mejorara significativamente el pronóstico ${ }^{2}$.

El objetivo del artículo es brindar una revisión general de las patologías con realce en anillo más frecuentes, las particularidades clínicas que hacen sospecharlas y las características del patrón observado mediante métodos diagnósticos mencionados, lo que permitirá al médico un abordaje más rápido y preciso de este tipo de patologías.

\section{MATERIALES Y MÉTODOS}

Se realizó una búsqueda sistemática en Bases de datos Internacionales (Pubmed, Embase, Lilacs, Cocrhaine), Bases de datos Latinoamericanas (Scielo, Redalic), y en Literatura gris en revistas nacionales. La selección de artículos se hizo en base a los buscadores en Mesh y Desc de cada una de las enfermedades a tratar en el artículo, asociado a los buscadores comunes NEUROIMAGEN (Neuroimaging) y DIAGNOSTICO (Diagnosis). El total de artículos encontrados fue de 1558 artículos.

Posteriormente, se aplican filtros de Tiempo (últimos 10 años), Humanos, Idioma (español, inglés, portugués), de lo cual se reduce a 764 artículos en total. De estos solo se seleccionan 70 , debido a que en ellos se encuentra especificado el principal patrón diagnostico por Neuroimagen común para las enfermedades: Patrón en anillo. Además, aportaron información importante como manifestaciones clínicas, y epidemiología. Los retratos fueron obtenidos del banco de imágenes del área de radiología e imágenes diagnosticas del Hospital San Rafael de Tunja, con previa autorización del comité ético y legal de dicha Institución.

\section{RESULTADOS}

\section{Toxoplasmosis cerebral}

Infección oportunista del SNC causada por la reactivación de una infección latente, previo contacto con el parasito ${ }^{6}$; esto ocurre especialmente en pacientes inmunocomprometidos (Síndrome de Inmunodeficiencia Adquirida (SIDA) o con trasplante de órganos) ${ }^{7}$. Sus formas de transmisión son el contacto directo con animales infectados, consumo de carnes contaminadas y transmisión vertical madre-hijo ${ }^{8,9}$. Se estima que aproximadamente la mitad de la población mundial ha estado en contacto con sepas de este parásito. La prevalecía en Estados Unidos es de $47 \%$ y $60 \%{ }^{10}$; en Colombia, predomina la sepa tipo I que presenta mayor agresividad y mecanismos de defensa ${ }^{10}$, por lo que su prevalencia es mucho mayor.

Manifestaciones clínicas:

Consisten en hidrocefalia, corioretinitis y calcificaciones cerebrales (Triada de Sabin). Sin embargo, rara vez se presentan en conjunto 9 . Pueden aparecer convulsiones tónico-clónicas, cefalea, hemiparesias, síndrome motor deficitario, síndrome cerebeloso, alteraciones de la memoria y la concentración, déficit focales en pares craneales; todos dependientes del número y localización de lesiones en el $\mathrm{SNC}^{11}$. El método de diagnóstico definitivo, se basa en estudios imagenológicos con TC y $\mathrm{RM}$, asociado a hallazgos serológicos y Biopsia ${ }^{12}$.

Diagnóstico por neuroimagen:

En la TC con contraste y en la RM, se observan lesiones intraparenquimatosas, únicas (15\%) o múltiples (85\%) (Figura 1) 12. Estas varían en tamaño desde microscópicas (3-9 milímetros), hasta lesiones de varios centímetros ${ }^{13}$; además presentan centro hipodenso y bordes resaltados compatibles con realce en anillo; en ocasiones lesiones hiperdensas, con realce solido cuando son pequeñas (Figura 1). Se comportan como lesiones con efecto masa asociadas a edema vasogénico circundante ${ }^{12,13}$ y de manera característica, las formas parasitarias se localizan en la periferia de las lesiones ${ }^{14}$. 
Sus localizaciones más frecuentes son los ganglios basales, regiones corticales y subcorticales de lóbulos temporal, frontal y parietal ${ }^{13}$. Tanto en la clínica como en la imagenología, la toxoplasmosis cerebral y el Linfoma tienen comportamiento similar, pero guardan algunas diferencias importantes (tabla 1).

Tabla 1. Diferencias imagenológicas de la Toxoplasmosis Cerebral y Linfoma para la realización de diagnóstico diferencial.

\begin{tabular}{|c|c|c|}
\hline CARACTERÍSTICA & TOXOPLASMOSIS & LINFOMA \\
\hline Tamaño de las lesiones & $<4 \mathrm{~cm}$ & $>4 \mathrm{~cm}$ \\
\hline Numero de lesiones & Múltiples & Solitarias/escazas \\
\hline Distribución & $\begin{array}{c}\text { Ganglios basales, interfase gris/ } \\
\text { blanca }\end{array}$ & Periventricular \\
\hline Hemorragia & + & $+/-$ \\
\hline Edema & +++ & + \\
\hline Realce & En anillo, de paredes delgadas & $\begin{array}{c}\text { Anillo de espesor irregular, } \\
\text { heterogéneo }\end{array}$ \\
\hline Efecto de masa & +++ & + \\
\hline Cuerpo calloso & Rara vez afectado & Afectado \\
\hline
\end{tabular}

\section{Linfoma primario del SNC}

Rara variante del linfoma tipo no hodgkin, con inicio en $\mathrm{SNC}^{15}$; es un tumor maligno que afecta ganglios basales, cuerpo calloso, tálamo, sustancia blanca periventricular y región subependimaria ${ }^{16-18}$. Corresponde al 1,5\% de todas las neoplasias intracraneales en pacientes inmunocompetentes y ocurre con mayor frecuencia en pacientes con SIDA. Afecta más a hombres, con relación $1,7: 1^{19,20}$.

Manifestaciones clínicas:

Se puede presentar focalidad neurológica $(70 \%)$, síntomas neuropsiquiátricos (43\%), hipertensión intracraneana (33\%), crisis epilépticas (14\%) y síntomas oculares (4\%) dentro de los que se destacan la visión borrosa, disminución de la agudeza visual, e inyección conjuntival ${ }^{21}$.

Estos tumores tienen rápida resolución con adecuado tratamiento, pero tienen tasa de recidiva muy alta durante el primer año $(90 \%)$, siendo el principal lugar de reaparición el mismo SNC, seguido de lugares extra cerebrales como cerebelo y meninges ${ }^{15}$.
Diagnóstico por neuroimagen:

El diagnóstico se basa en la biopsia cerebral. Esta técnica resulta costosa y restringida, razón por la cual se realiza la TC y la RM. En el caso de la TC simple las lesiones son isodensas o hiperdensas ( $92 \%$ ), pero después de la administración de contraste manifiestan reborde hiperdenso con centro hipodenso no asociados a edema vasogénico compatible con el patrón de realce en anillo ${ }^{22}$ (Figura 1); en la RM adquiere un patrón de isointensidad o hipointensidad en T2, debido a la alta relación núcleo/ citoplasma de las células malignas ${ }^{18}$. Es de tener en cuenta que la presencia de una lesión única, de gran tamaño ( $>3 \mathrm{~cm}$ ) y localizada a nivel de la sustancia blanca periventricular sugiere el diagnóstico de Linfoma ${ }^{12,16,23}$.

\section{Metástasis en SNC}

Es la complicación más frecuente en los pacientes oncológicos ${ }^{24}$; así mismo, es la neoplasia más frecuente del $\mathrm{SNC}^{25}$.

Tienen como origen el cáncer de pulmón (50\%), cáncer de mama (15-20\%), el melanoma (10\%) y con menor frecuencia el cáncer renal y colo-rectal; de 
estos, las metástasis cerebrales provenientes de cáncer de seno, colon y renal son a menudo únicas. Las provenientes de melanoma y el cáncer de pulmón tienen a ser multifocales ${ }^{25,26}$. Las metástasis se localizan en los hemisferios cerebrales $(80 \%)$, cerebelo $(15 \%)$, tronco cerebral (5\%) y muy poco en ganglios basales ${ }^{26}$.

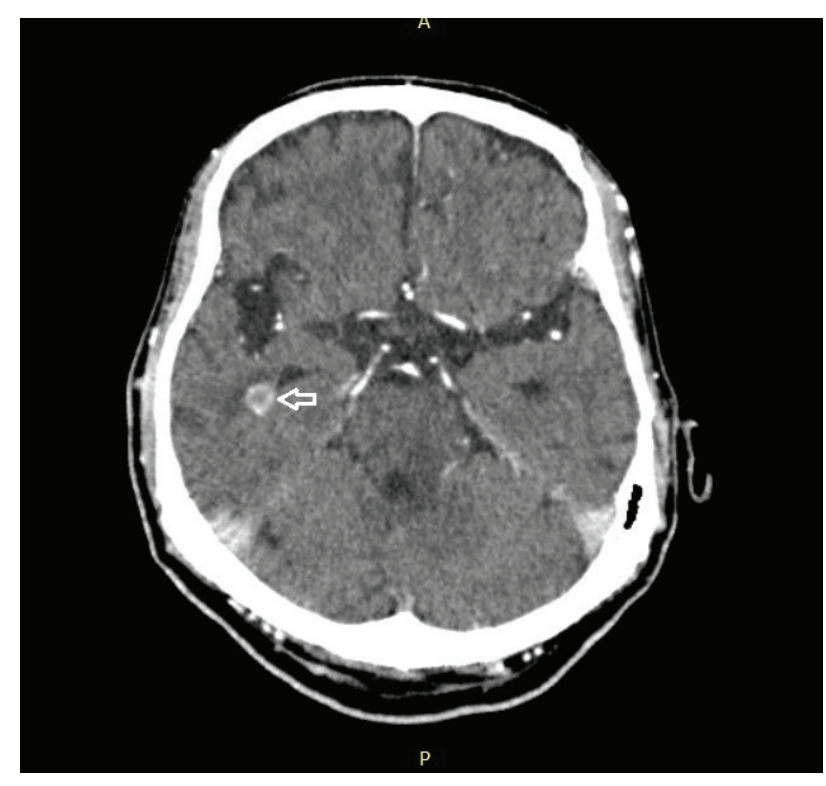

Figura 1: TC con contraste. Lesión en lóbulo temporal derecho, que luego de la administración de contraste toma realce en anillo (flecha blanca). Se acompaña de una zona hipodensa alrededor de la lesión, compatible con edema vasogénico. Debido al comportamiento de la lesión; se tiene como diagnósticos diferenciales toxoplasmosis, neurocisticercosis y linfoma primario del SNC.

El diagnóstico aparece en pacientes con neoplasia ya diagnosticada previamente (60-80\%), con un tiempo de aparición de 12 meses; en el 20-30\% aparece al mismo tiempo con el diagnóstico del cáncer primario ${ }^{24,26}$. La edad más frecuente de aparición es de los 55 a 65 años y la incidencia es de 100.000 a 170.000 nuevos casos por año en los Estados Unidos ${ }^{27}$.

\section{Manifestaciones clínicas:}

Dependen de la localización de la(s) lesión(es) y los síntomas son similares a los producidos por tumores primarios del $\mathrm{SNC}^{27}$. Se diferencian por el predominio de síntomas difusos de las metástasis dados por el edema vasogénico, lesiones de gran volumen con efecto masa y lesiones múltiples ${ }^{26}$, por lo que se presenta disfunción cognitiva, alteraciones mentales, cefalea, déficit neurológico, hemiparesias, afasia y alteraciones visuales; con menor frecuencia náuseas y vómito, sincope y papiledema ${ }^{28}$. Las hemorragias dentro de las lesiones metastásicas pueden producir cefalea intensa de inicio súbito, y llevar a estado de coma ${ }^{28}$.

\section{Diagnóstico por neuroimagen:}

En la TC y RM las metástasis cerebrales se clasifican según el número de lesiones en: i) únicas, ii) oligometástasis (una y tres lesiones) iii) y múltiples (más de tres lesiones) ${ }^{28}$; estas producen efecto masa con desplazamiento de estructuras vecinas afectando espacios subaracnoideos 0 ventriculares, dependiendo la ubicación ${ }^{12}$; se localizan en la unión entre la sustancia gris y blanca, y con contraste, se produce intensificación de los bordes de la lesión, en forma de anillo (Figura 2) ${ }^{28}$.

\section{Neurocisticercosis:}

Parasitosis producida por larvas de Taenia solium enquistadas en SNC adquirida por ingerir alimentos contaminados con sus huevos ${ }^{29-31}$ siendo la causa más común prevenible de epilepsia ${ }^{31,32}$. Según la OMS se conoce que más del $80 \%$ de los 50 millones de personas afectadas en el mundo por epilepsia viven en lugares donde predomina esta infección ${ }^{33}$; en América latina, se ha descrito la existencia de neurocisticercosis en 18 países, aproximadamente 350.000 pacientes infectados ${ }^{30}$. En Colombia, se han documentado casos en Antioquia, Nariño y Valle del Cauca, mediante estudios serológicos pero no hay estudios de prevalencia ${ }^{33}$.

\section{Manifestaciones Clínicas:}

Depende de la localización de las lesiones en el SNC. Como se mencionó, es una de las principales causas de epilepsia adquirida, y las lesiones se observan en regiones subaracnoidea o ventricular desde donde se generan signos de focalización neurológica $(20 \%)^{34,35}$; también se afecta el tracto piramidal, presentándose déficit sensorial, de lenguaje, movimientos involuntarios y rigidez muscular. Los síndromes isquémicos en cápsula o región talámica son raros (3\%), manifestadas por pérdida de la conciencia, disminución de agudeza visual, cefalea, vómito y papiledema $^{36}$; en casos severos ocurre demencia ${ }^{37}$.

El tratamiento va desde cesticidas, corticosteroides hasta la resección quirúrgica de la calcificación ${ }^{38,39}$.

Diagnóstico por neuroimagen: 
La TC y la RM son los métodos diagnósticos de elección ${ }^{30}$, y en ellos se evidencian los siguientes estadios:

1. Estadio no quístico: asintomático, corresponde a la invasión del cisticerco. Se observa foco localizado de edema, puede tener realce en anillo tras la administración de contraste

2. Estadio vesicular: inicio de la respuesta inmune con mínima inflamación. Se observa quiste redondeado con una cápsula fina (larva viable, vesícula ocupada por líquido)

3. Estadio vesicular-coloidal: parásitos muertos que desencadenan reacción inflamatoria aguda. Se asocia a edema perilesional y la formación de una cápsula hipodensa. Con contraste se observa realce en anillo (Figura 1)

4. Estadio nodular-granulomatoso: retracción del quiste, su cápsula es gruesa y el escólex se calcifica. La TC muestra un quiste isodenso con un escólex calcificado hiperdenso

5. Estadio nodular-calcificado: lesión mineralizada. En la TC se observa nódulo calcificado sin efecto de masa ni captación de contraste ${ }^{24,32,35,40}$ the most common form encountered by physicians, is the use of both albendazole and praziquantel as a treatment strategy. Patients with subarachnoid NCC (SANCC.

Asociado a lo anterior, la RM es muy sensible para observar lesiones muy pequeñas y quistes interventriculares ${ }^{34}$.

\section{Glioblastoma multiforme (GBM)}

Astrocitoma maligno Grado IV que presenta una alta frecuencia en el adulto ${ }^{41,42}$, siendo responsable del $70 \%$ de todos los gliomas y $20 \%$ de todos los tumores intracraneales ${ }^{43}$. Su pronóstico es desfavorable por su alto grado de malignidad ${ }^{44}$; esto asociado a que no se conoce su causa con exactitud ${ }^{45,46}$. Predomina en hombres $(3: 2)$, entre 50 y 60 años, con sobrevida aproximada de 9 a 12 meses $^{47,48}$, y la incidencia en Norteamérica y Europa es de 2 a 3 casos por 100.000 habitantes $^{48}$.

\section{Manifestaciones clínicas:}

Sus síntomas son muy inespecíficos dados por cefalea, náuseas, emésis, vasodilatación cerebral y periférica, alteración del estado de conciencia, alteraciones retinianas (papiledema), hemiparesias y crisis convulsivas ${ }^{41,49}$, siendo los sitios más afectados son los lóbulos temporal, parietal y frontal ${ }^{50}$.
Diagnóstico por neuroimagen:

La RM tiene mayor sensibilidad y especificidad ${ }^{51}$, pero en ambos métodos se observan tres patrones característicos: necrosis, hemorragia y neovascularización. Se observa masa única con necrosis central, intensificación en anillo (células con alta actividad proliferativa) y un área peritumoral extensa de edema vasogénico (Figura 2).

En la RM se observan hipointensidad en T1 e hiperintensidad en T2. Puede presentar diseminación bihemisférica a través del cuerpo calloso, con afección de lóbulos frontales (Glioma en "alas de mariposa") ${ }^{50,52}$.

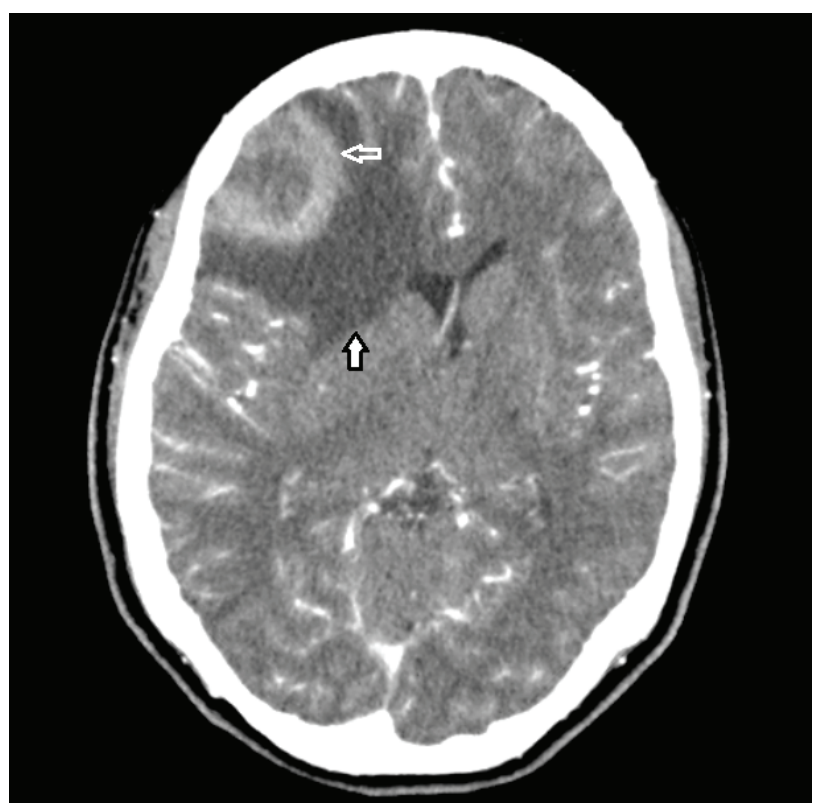

Figura 2: TC con contraste. Lesión ocupante de espacio con efecto masa ubicada en la región frontotemporal derecha. Se aprecia la lesión con centro hipodenso y bordes con realce en forma anular (flecha blanca). Así mismo se observa una zona hipodensa alrededor de la lesión correspondiente a edema vasogénico (flecha negra); se tiene como diagnósticos diferenciales tumor primario en SNC (Glioblastoma multiforme) o metástasis.

\section{Absceso cerebral}

Proceso inflamatorio focal intraparenquimatoso, dado por la acumulación de material purulento dentro de una cápsula vascularizada que tiene efecto masa intracraneal. 
Las vías de infección más frecuentes son la extensión de un foco pericraneal (otitis, sinusitis o infección dentaria) $(50 \%)^{53}$, la diseminación hematógena desde un foco distal de infección (absceso pulmonar, infecciones intrabdominales) (15 -30\%) o secundario a trauma craneoencefálico o cirugía $(8-19 \%)^{54,55}$.

Se conocen cuatro etapas del proceso: a) inicia con la cerebritis temprana que va desde el primer al tercer día, en la cual el foco necrótico es poco definido con inflamación periférica; b) la cerebritis tardía que ocurre del cuarto al noveno día, en donde el área de necrosis aumenta de tamaño; c) la capsular temprana que ocurre del décimo al decimotercer día, en la cual inicia la formación de capsula fibrosa rodeada de inflamación perivascular; d) finalmente la capsular tardía donde a partir del decimotercer día, finaliza el proceso de maduración del absceso, se reduce el área de necrosis y disminuye la inflamación periférica ${ }^{56}$.

La incidencia anual del absceso cerebral es de 1500 a 2000 casos en América, con mayor frecuencia en países subdesarrollados (4 a 11 casos por cada millón de habitantes) ${ }^{57}$; predomina en hombres, con una relación variable de 3:1 y generalmente se presenta en las 2 primeras décadas de la vida ${ }^{58}$.

\section{Manifestaciones clínicas:}

Sus síntomas son inespecíficos siendo los más frecuentes cefalea (49- 93\%), fiebre (14-88\%) y alteración del estado mental (29\%); en conjunto solo se presenta en un $20 \%$ de los $\operatorname{casos}^{59,60}$.

\section{Diagnóstico por neuroimagen:}

La TC es el método de elección, con una sensibilidad del $95 \%$ y especificidad del $85 \%$. En las etapas de cerebritis temprana y tardía, se observa lesión con centro hipodenso, mientras que en las etapas capsular temprana y tardía, se aprecia un patrón en anillo, el cual está dado por un centro hipodenso y una cápsula hiperdensa, que resalta con medio de contraste ${ }^{56}$ (Figura 3).

En la RM, durante las fases tanto temprana como tardía, las secuencias T1 muestran una imagen hipointensa y en T2 hiperintensa, ambas lesiones con contornos borrosos; en la fase capsular temprana, se observa capsula hiperintensa y un centro hipointenso en T1 mientras que en $\mathrm{T} 2$ permanecen ambos elementos hipointensos; finalmente, en la etapa capsular tardía, en T1 la capsula se muestra hiperintensa y el centro hipointenso; por lo contrario, en T2 la capsula es hipointensa y el centro hiperintenso $0^{56,61}$.

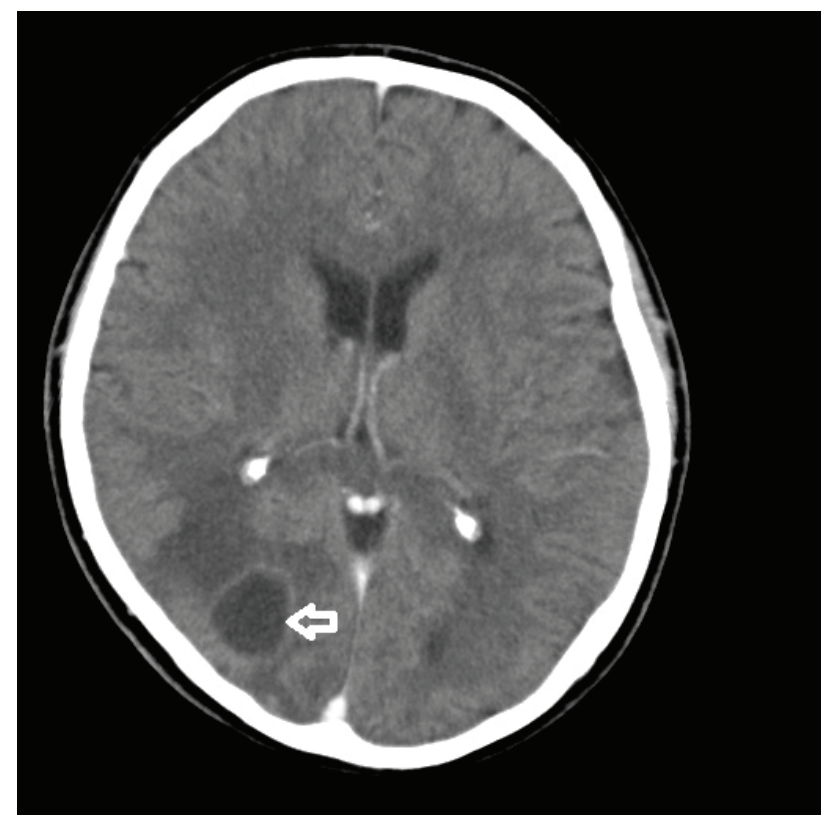

Figura 3: TC simple. En la imagen se aprecia una lesión en la región occipital derecha, con centro hipodenso bien delimitada dentro de una cápsula hiperdensa (flecha blanca), la cual corresponde a un absceso cerebral en etapa capsular tardía.

\section{Enfermedad Desmielinizante}

Comprende un grupo de patologías caracterizadas por la afección y destrucción de las vainas de mielina sanas. La más frecuente de este grupo es la Esclerosis Múltiple $(\mathrm{EM})^{62}$, siendo esta una enfermedad desmielinizante crónica, autoinmune e inflamatoria, cuyos factores de riesgo más frecuentes incluyen las infecciones virales (Epstein-Barr), el tabaquismo, poca o nula exposición a la luz solar y la deficiencia de Vitamina $\mathrm{D}^{62,63}$. Se caracteriza por inflamación perivenosa, desmielinización y gliosis ${ }^{63}$.

Es la enfermedad neurológica crónica más frecuente en adultos jóvenes de Europa y Norteamérica, predominante en raza blanca, y poco común en países tropicales y subdesarrollados; en Latinoamérica, la prevalencia es de 2 a 13 casos por cada 100.000 habitantes, siendo en Cuba y México un poco mayor, en una proporción de 103 casos por cada 100.000 habitantes $^{64}$.

\section{Manifestaciones Clínicas}

Las manifestaciones clínicas más frecuentes son trastornos sensitivos y motores en una o más extremidades ( $>50 \%$ casos), neuritis óptica ( $25 \%$ casos), ataxia, vejiga 
neurogénica, fatiga, disartria, nistagmo, vértigo $(<10 \%$ casos), afasia, apraxia, convulsiones o demencia, y los signos extrapiramidales $(59,60)^{61,63}$.

\section{Diagnóstico por Neuroimagen:}

La TC es poco sensible y normalmente no muestra ninguna alteración. La RM es la técnica más sensible y específica en la identificación de lesiones desmielinizantes del SNC, principalmente en EM, y es más específica cuando se contrasta con gadolinio ${ }^{62}$; en las secuencias $\mathrm{T} 2$ con ganodilio se evidencia hiperintensidad en todo el tejido cerebral, que refleja una ruptura inflamatoria de la barrera hematoencefálica y aumento tisular del agua libre. Las lesiones iniciales usualmente aparecen como nódulos de tamaño menor a $25 \mathrm{~mm}$, la cuales pueden evolucionar a formas en anillo o de arco y se localizan principalmente en tejido parenquimatoso cerebral con una distribución perivascular; estas lesiones pueden confluir, formando placas hiperintensas con bordes irregulares, con forma característica en sierra (35\%). Se localizan con mayor frecuencia en sustancia blanca periventricular $(60 \%)$, cuerpo calloso $(25 \%)$, sustancia blanca yuxtacortical y parénquima infratentorial $(15 \%)^{61,65}$.

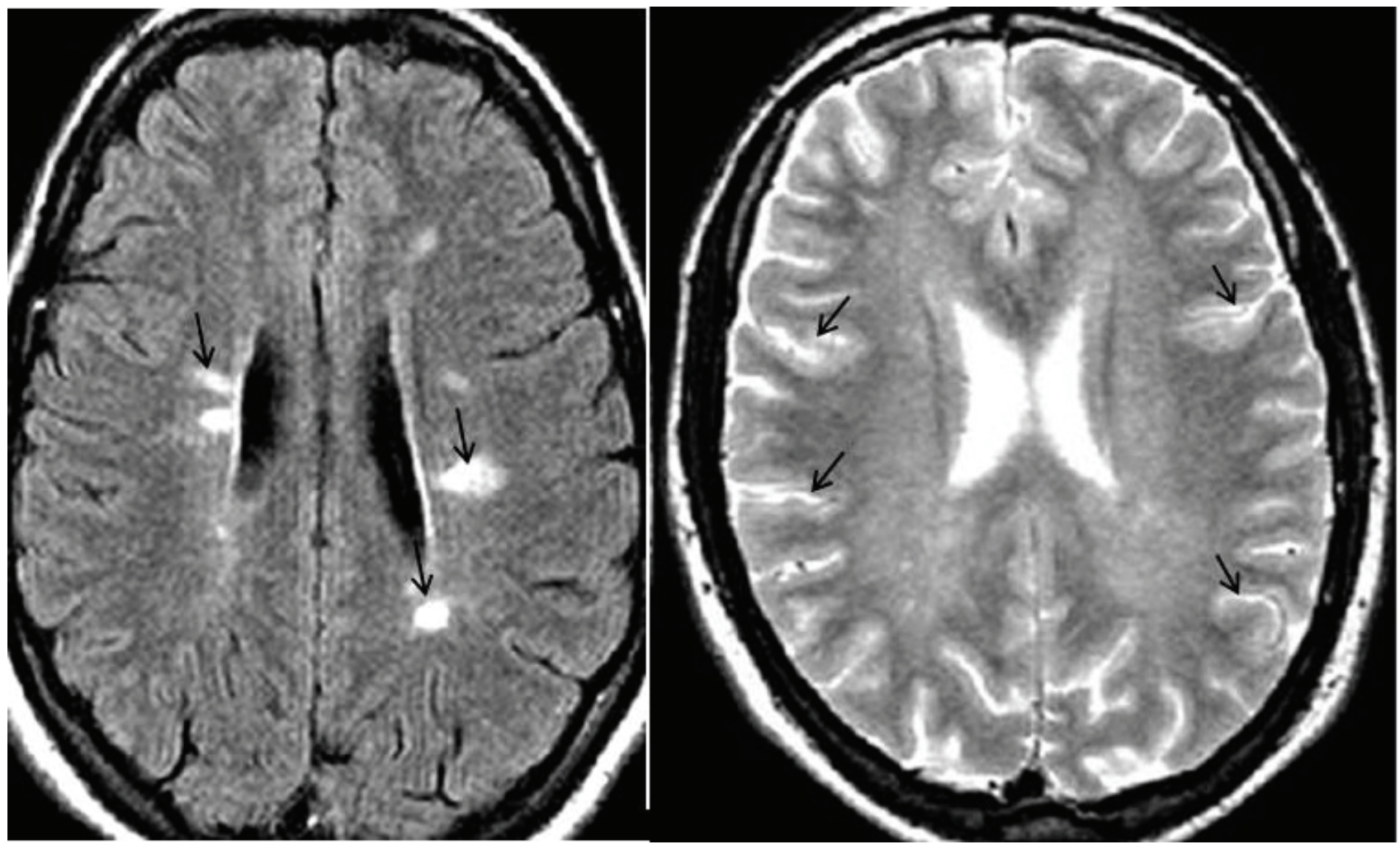

Figura 4: a la derecha RM corte T1 que muestra lesiones intraparenquimatosas periventriculares hiperintensas. se aprecia patrón en sierra en lesiones cercanas el ventrículo lateral derecho (flechas negras). A la Izquierda se evidencia Lesiones yuxtacorticales en secuencia FLAIR (flechas negras)

\section{DISCUSIÓN}

En la actualidad los métodos de diagnóstico radiológico suponen una ayuda al clínico en la resolución de problemas de la profesión médica ${ }^{1,3}$; esto principalmente en la diferenciación de patologías que afectan el SNC, con lo que se destaca la participación de la TC simple y con contraste y la RM como las principales ayudas radiológicas de las que se dispone en el diagnóstico de las patologías antes mencionadas ${ }^{3,4,12}$.

Como se ha mencionado, el patrón radiológico en anillo en SNC en la TC y RM se presenta en múltiples patologías, por lo cual se hace necesario la identificación de las particularidades clínicas y paraclínicas de cada una de las enfermedades que presentan este patrón ${ }^{12,22,28,40}$. Un 
adecuado abordaje diagnóstico incluye la realización de una historia clinica detallada, donde los antecedentes exposicionales, la evolución clínica de la enfermedad y los hallazgos en el examen físico aportan información valiosa y especifica que ayuda al clínico en un adecuado diagnóstico y por ende en un adecuado tratamiento ${ }^{12,51,52}$. Además, debido a la similitud en el patrón imagenológico de estas enfermedades, se hace necesaria la ayuda de otros métodos y ayudas diagnósticas que junto con la sospecha clinica, permitan confirmar o descartar dichas enfermedades ${ }^{7,16,25,35,55,65}$. Para lo anterior proponemos un flujograma guía de diagnóstico clínico y paraclínico, que permitirá al médico un mejor abordaje del paciente cuando presente este tipo de patrón radiológico (Figura 4). Insistimos en que, del adecuado abordaje diagnóstico de estas enfermedades neurológicas, y que por su alta morbimortalidad, dependerá el pronóstico y la calidad de vida de los pacientes.

\section{REFERENCIAS BIBLIOGRÁFICAS}

1. Motta G, Arroyo G, Quiroz O, Ramírez J. Impacto de la tomografía computada de multidetectores (TCMD) en la práctica médica. Evaluación retrospectiva de solicitudes y diagnósticos por TCMD. Acta medica grupo los Angeles, 2010; 6(2): 55-63.

2. Bastarrika G. Tomografía computarizada y práctica clínica. An. Sist. Sanit. Navar, 2007; 30:171-176.

3. Mendizábal A. Radiación ionizante en tomografía computada: un tema de reflexión. Anales de Radiología México, 2012; 2:90-97.

4. Gálvez M, Bravo C, Rodríguez C, Farías A, Cerda C. Características De Las Hemorragias Intracraneanas Espontaneas En TC Y RM. Rev Chil Radiología, 2007; 13(1):12-25.

5. Fica C, Bustos G, Miranda C. Absceso cerebral: A propósito de una serie de 30 casos. Rev Chil infectología. 2006; 23(2):140-9.

6. Rosso F, Agudelo A, Isaza Á, Montoya J. Congenital toxoplasmosis: Clinical and epidemiological aspects of the infection during pregnancy. Colombia Médica, 2007; 38(3): 316-37.

7. Sakamoto N, Maeda T, Mikita Y, Kato Y, Yanagisawa N, Suganuma A, et al. Clinical presentation and diagnosis of toxoplasmic encephalitis in Japan. Parasitol Int, 2014; 63(5):701-4.

8. Santa I, Valbuena Y, Cortes L, Sánchez A. Seroprevalencia de la toxoplasmosis y factores relacionados con las enfermedades transmitidas por alimentos en trabajadores de plantas de beneficio animal en cinco ciudades capitales de Colombia, 2008. NOVA-Publicación científica en ciencias biomédicas, 2009; 7(11): 66-70.
9. Contini C. Clinical and diagnostic management of toxoplasmosis in the immunocompromised patient. Parassitologia, 2008; 50(1-2):45-50.

10. Morisset S, Peyron F, Lobry JR, Garweg J, Ferrandiz $\mathrm{J}$, Musset K, et al. Serotyping of Toxoplasma gondii: striking homogeneous pattern between symptomatic and asymptomatic infections within Europe and South America. Microbes Infect. 2008; 10(7):742-7.

11. Correia C, Melo H, Costa V, Brainer A. Features to validate cerebral toxoplasmosis. Rev Soc Bras Med Trop, 2008; 46(3):373-6.

12. Miranda G, Díaz C, Dellien H, Hermosilla H. Enfrentamiento Imagenológico de las lesiones cerebrales en pacientes VIH. Rev Chil Radiol; 2008; 14(4):200-7.

13. Ramírez M, Varela MA, Aranza JL, García A, Colunga J, Jiménez M, Rodriguez M. toxoplasmosis cerebral y SIDA en un adolescente, 2014; 30: 204-208.

14. Madero G, Cerquera F, Borrero L. Toxoplasmosis cerebral congénita: reporte de un caso. Rev Colomb Radiol. 2009; 20(4):2784-8

15. Zaspe I, Llano P, Gorosquieta A, Cabada T, Muñón T, Vázquez A, et al. Linfoma cerebral primario: revisión bibliográfica y experiencia en el Hospital de Navarra en los últimos 5 años (2000-2004). An Sist Sanit Navar, 2009; 28(3):367-77.

16. Castro-Rebollo M, Vleming EN, Drake-Rodríguez P, Benítez-Herreros J, Pérez-Rico C. Diagnóstico de linfoma cerebral primario por el oftalmólogo. Arch Soc Esp Oftalmol, 2010; 85(1):35-37.

17. Commins D. Pathology of primary central nervous system lymphoma. Neurosurg Focus, American Association of Neurological Surgeons; 2006; 21(5):1-10.

18. Málaga J, Mamani JA, Fuentes M, Suclla J, Meza J. Linfoma primario del sistema nervioso central en un paciente inmunocompetente. An la Fac Med UNMSM. Facultad de Medicina, 2013; 73(3):245-50.

19. Arteaga C, Duarte M, Bayona H, Andrade R, López R, Bermúdez S. Linfoma cerebral en paciente postrasplante renal. Acta Med Colomb, 2009; 34(1): 33-37.

20. Alécio-Mattei T, Alécio-Mattei J, Aguiar P, Ramina R. Primary central nervous system lymphomas in immunocompetent patients. Neurocirugia (Astur). 2006; 17(1):46-53.

21. Ferreri AJM. How I treat primary CNS lymphoma. Blood 2011; 118(3):510-22.

22. Cabrera S, Krygier G, Dutra A, Sosa A, Lombardo K, Savio E, et al. Linfoma primario del sistema nervioso central en un paciente con sida. Rev Med Uruguay 2005; $21: 68-74$

23. Troiani C, Lopes C, Scardovelli C, Nai G. Cystic brain metastases radiologically simulating neurocysticercosis. Sao Paulo Med J, 2011; 129(5):352-6. 
24. Nieder C, Spanne O, Mehta MP, Grosu AL, Geinitz H. Presentation, patterns of care, and survival in patients with brain metastases: what has changed in the last 20 years? Cancer. 2011; 117(11):2505-12.

25. Norden AD, Wen PY, Kesari S. Brain metastases. Current Opinion in Neurology, 2005; 18:654-661.

26. Lovo I, Torrealba M, Villanueva G, Gejman R, Tagle M. Metástasis cerebral y sobrevida. Rev. méd. Chile, 2005; 133(2):190-194.

27. Rodríguez M, Villafuerte D, Conde T, Díaz Y, Martínez A, Rivero CR. Caracterización tomográfica e histológica de las neoplasias intracraneales. MediSur, 2010; 8(2):9-14.

28. Andreia S, Veiga A. Conus Terminallis Neurocysticercosis: A Rare Cause of Lumbar Radiculopathy. J Neurol Neurophysiol, 2015; 6:1.

29. Saavedra H, Gonzales I, Alvarado MA, Porras MA, Vargas V, Cjuno RA, et al. Diagnóstico y manejo de la neurocisticercosis en el Perú. Rev Peru Med Exp Salud Pública. 2010; 27(4):586-91.

30. Baird RA, Wiebe S, Zunt JR, Halperin JJ, Gronseth G, Roos KL. Evidence-based guideline: treatment of parenchymal neurocysticercosis: report of the Guideline Development Subcommittee of the American Academy of Neurology. Neurology, 2013; 80(15):1424-9.

31. Del Brutto OH. Diagnostic criteria for neurocysticercosis, revisited. Pathog Glob Health, 2012; 106(5):299-304.

32. Flórez AC, Pastrán SM, Peña PA, Benavides A, Villareal A, Rincón C, et al. Cisticercosis en Boyacá, Colombia: estudio de seroprevalencia, Acta Neurol Colomb, 2011; 27(1):9-18.

33. Del Brutto O. Neurocysticercosis: a review. Scientific World Journal. 2012;2012.

34. Coyle CM. Neurocysticercosis: an update. Curr Infect Dis Rep, 2014; 16(11):437.

35. Nash TE, Pretell EJ, Lescano AG, Bustos JA, Gilman RH, Gonzalez AE, et al. Perilesional brain edema and seizure activity in patients with calcified neurocysticercosis: a prospective cohort and nested case-control study. Lancet Neurol, 2008; 7(12):1099-105.

36. Fleury A, Carrillo-Mezo R, Flisser A, Sciutto E, Corona T. Subarachnoid basal neurocysticercosis: a focus on the most severe form of the disease. Expert Rev Anti Infect Ther, 2011; 9(1):123-33

37. Nogales J, Arriagada R, Salinas R. Tratamiento de la neurocisticercosis: Revisión crítica. Rev Med Chil Sociedad Médica de Santiago, 2006; 134(6):789-96.

38. Sombert E, Fong J, González R. Diagnóstico y tratamiento de la neurocisticercosis en una mujer joven. MEDISAN, 2014; 18(2): 271-5.

39. Sarria S, Frascheri L, Siurana S, Auger C, Rovira A. Imaging findings in neurocysticercosis. Radiologia, 2013; 55(2): 130-41.

40. Louis DN, Ohgaki H, Wiestler OD, Cavenee WK, Burger PC, Jouvet A, et al. The 2007 WHO classification of tumours of the central nervous system. Acta Neuropathol, 2007; 114(2):97-109.

41. Sathornsumetee S, Rich J, Reardon D. Diagnosis and treatment of high-grade astrocytoma. Neurol Clin, 2007; 25(4):1111-39.

42. Rodríguez R, Lombardo K, Roldán G, Silvera J, Lagomarsino R. Glioblastoma multiforme cerebral hemisférico Análisis de sobrevida de 65 casos tratados en el Departamento de Oncología del Hospital de Clínicas desde 1980 a 2000. Rev Med Urug, 2012; 28:250-261

43. Tait M, Petrik V, Loosemore A, Bell B, Papadopoulos M. La supervivencia de los pacientes con glioblastoma multiforme no ha mejorado entre 1993 y 2004: análisis de 625 casos. Br J Neurosurg, 2007; 21(5):496-500.

44. Vrrell C, Plotkin S. Las causas genéticas de los tumores cerebrales: la neurofibromatosis, esclerosis tuberosa, enfermedad de von Hippel-Lindau, y otros síndromes. Neurol Clin, 2007; 2(4):925-46.

45. Rees J, Smirniotopoulos J, Jones R, Wong K. Glioblastoma multiforme: radiologic-pathologic correlation. Radiographics. 1996; 16(6):1413-38; quiz 1462-3.

46. Hernández-Reyna R, Medellín-Sánchez R, Cerda-Flores RM, Calderón-Garcidueñas AL. Factores pronósticos de supervivencia en pacientes mexicanos con glioblastoma multiforme. Rev Med Inst Mex Seguro Soc, 2010; $8(2): 121-6$.

47. Blake L, Maravilla K, Berger M, Wilson C. Computed Tomografhy In The gliomas. Philadelphia: WB Saunders, 2005; 2(2): 333-347.

48. Schwartzbaum J, Fisher J, Aldape K, Wrensch M. Epidemiology and molecular pathology of glioma. Nat Clin Pract Neurol, Nature Publishing Group, 2006; 2(9):494-503.

49. Kanu O, Mehta A, Di C, Lin N, Bortoff K, Bigner DD, et al. Glioblastoma multiforme: a review of therapeutic targets. Expert Opin Ther Targets. 2009; 13(6):701-18.

50. Guerrero T, López I, Cartier L. Glioma multifocal multicéntrico. Rev Chil Neuropsiquiatr, 2010; 48(3):213-8.

51. Brant W, Helms C. Fundamentos de radiologia Diagnóstico por imagenes. 3 ed. Rio de Janeiro: Guanabara Koogan, 2008.

52. Kerschensteiner M, Stadelmann C, Dechant G, Wekerle $\mathrm{H}$, Hohlfeld R. Neurotrophic cross-talk between the nervous and immune systems: implications for neurological diseases. Ann Neurol, 2003; 53(3): 292-304.

53. Gómez J, García E, Martínez M, Martínez J, Gonzalez J, Pérez MA, et al. Abscesos cerebrales: análisis de factores pronóstico e influencia del tratamiento antibiótico protocolizado en su evolución. An Med Interna, 2008; 25:331-4.

54. Céspedes M, Choque F, Díaz S, Melgar N. Absceso cerebral, a propósito de un caso. Rev la Soc Boliv Pediatría, 2009; 48(2):92-5. 
55. Alvis H, Castella SM, Elzain MA, Moscote LR. Brain abscess: Current management. J Neurosci Rural Pract, 2013; 4:67-81.

56. Molina G. Armijo E. Mimenza A. Absceso cerebral. Rev Mex Neurocienc, 2010; 11(1):63-70.

57. Gutiérrez M, Ballesteros MA, Vallejo A, Miñambres E, Fariñas C, García JD, et al. Abscesos cerebrales en un hospital de tercer nivel: epidemiología y factores que influyen en la mortalidad. Rev Española Quimioter. 2009; 22(4):201-6.

58. Muzumdar D, Jhawar S, Goel A. Brain abscess: an overview. Int J Surg Elsevier, 2011; 9(2):136-44.

59. Orozco A, Monsalve D. Brain Abscess From Escherichia Coli: A Simple Diarrhea, Doctor? Rev Med Universidad Militar Nueva Granada, 2008; 16(2):243-8.

60. Patel K, Clifford D. Bacterial brain abscess. The Neurohospitalist 2012; 4(4):196-204.

61. Sarrazin J, Bonneville F, Martin G. Brain infections. Diagn Interv Imaging, 2012; 93(6):473-90.
62. Bester M, Petracca M, Inglese M. Neuroimaging pf Multiple Sclerosis, Acute disseminated Encephalomyelitis, and other Demyelinating Diseases. Seminars in Roentgenology, 2014; 49 (1): 76-85.

63. Macchi B, Marino-Merlo F, Nocentini U, Pisani V, Cuzzocrea S, Grelli S et al. Role od inflammation and apoptosis in multiple sclerosis: comparative analysis between the periphery and the central system. Journal of Neuroinmunology, 2015; 287: 80-87.

64. Domínguez Moreno R, Morales Esponda M, Rossiere MN, Triano RO, Gutierrez JL. Esclerosis Múltiple: revisión de la literatura médica. Revista de la faculta de Medicina de la UNAM, 2012; 55 (5): 226-33.

65. Sethi V, Yousry TA, Muhlert N, Ron M, Golay X, WheelerKingshott $C$ et al: Improved detection of cortical MS lesions with phase sensitive inversion $\mathrm{r}$ ecovery MRI. J Neurol Neurosurg Psychiatr, 2012; 83(9):877-882.

Para citar este artículo: Aljure V, Pulido-Arias E, Rodríguez-Monroy J, Rodríguez-Mateus M, Ramos-Hernández M. Diagnóstico diferencial de lesiones cerebrales con realce en anillo en tomografía computarizada y resonancia magnética. Duazary. 2016 julio; 13 (2): 149 - 158 